\title{
Phase Separation in the Two-Dimensional Systems of Strongly Correlated Electrons; the Role of Spin Singlet Pairs on Hole Pairing Contribution to Hole-rich Phase
}

\author{
Sung-Sik Lee, Sul-Ah Ahn, and Sung-Ho Suck Salk ${ }^{1,2}$ \\ 1 Department of Physics, Pohang University of Science and Technology, Pohang 790-784, Korea \\ ${ }^{2}$ Korea Institute of Advanced Study, Seoul 130-012, Korea
}

(October 24, 2018)

By paying attention to the hole-doped two-dimensional systems of antiferromagnetically (strongly) correlated electrons, we discuss the cause of hole-rich phase formation in association with phase separation. We show that the phase diagram obtained from the Maxwell's construction in the plane of temperature vs. hole density is consistent with one derived from the evaluation of hole-rich and electron-rich phases in real space. We observe that the formation of a hole-rich phase is attributed to the aggregation of hole pairs induced by spin singlet pairs present in the pseudogap phase and that a direct involvement of correlations between hole pairs are not essential for phase separation.

PACS numbers: 64.75.+g, 71.10.Hf, 73.22.Gk, 74.80.-g

Currently there has been an activated interest in the study of stripe modulations owing to strong experimental evidences observed [1 13] involving hole-doped high $T_{c}$ cuprates. Phase separation, a phenomenon of broken symmetry at low energies can be manifested as a stripe phase or a phase-separated inhomogeneity. At a critical temperature, the phase separation appears owing to the divergence of compressibility or the zero inverse compressibility. The onset of phase separation can be obtained from the evaluation of the zero inverse compressibility using the well-known Maxwell's construction. Thus far, various numerical approaches [14 32] to the Hubbard and $t-J$ Hamiltonians have successfully revealed the existence of phase separation in the hole-doped two dimensional systems of strongly correlated electrons. They include the Hatree-Fock (HF), the Lanczos exact diagonalization (LED), the density matrix renormalization group (DMRG), a fixed node quantum Monte Carlo (FNQMC) and Green's function Monte Carlo (GFMC) methods. Most recently a slave-boson functional integral (SBFI) method of Gimm and Salk [30] has been proposed to reveal a good agreement with earlier exact numerical studies [15,25] of phase separation boundary. However it remains to be seen whether the phase separation obtained from the Maxwell's construction can, indeed, show phaseseparated distributions of charge and spin in real space. Currently there exists a great controversy [31,32, 21,25] over the issue of whether the phase separation accompanied by the hole-rich phase arises as a consequence of correlations between pairs of holes or not. Here we address this issue, based on our slave-boson functional integral approach [30,33], and take the advantage of its usefulness to study the role of charge and spin degrees of freedom on phase separation.

The $t-J$ Hamiltonian to deal with the hole doped systems of antiferromagnetically correlated electrons is written,

$$
\begin{aligned}
H= & -t \sum_{\langle i, j\rangle \sigma}\left(c_{i \sigma}^{\dagger} c_{j \sigma}+\text { H.c. }\right)+J \sum_{\langle i, j\rangle}\left(\mathbf{S}_{i} \cdot \mathbf{S}_{j}-\frac{n_{i} n_{j}}{4}\right) \\
= & -t \sum_{\langle i, j\rangle \sigma}\left(c_{i \sigma}^{\dagger} c_{j \sigma}+\text { H.c. }\right) \\
& -\frac{J}{2} \sum_{\langle i, j\rangle}\left(c_{i \uparrow}^{\dagger} c_{j \downarrow}^{\dagger}-c_{i \downarrow}^{\dagger} c_{j \uparrow}^{\dagger}\right)\left(c_{j \downarrow} c_{i \uparrow}-c_{j \uparrow} c_{i \downarrow}\right) .
\end{aligned}
$$

In order to explicitly see physics involved with the spin and charge degrees of freedom, we rewrite the above equation in the $U(1)$ slave-boson representation with the use of $c_{i \sigma}=f_{i \sigma} b_{i}^{\dagger}$ as a composite of spinon $f_{i \sigma}$ and holon $b_{i}$ operators subject to the single occupancy constraint $b_{i}^{\dagger} b_{i}+\sum_{\sigma} f_{i \sigma}^{\dagger} f_{i \sigma}=1$

$$
\begin{aligned}
H_{t-J}= & -t \sum_{\langle i, j\rangle \sigma} f_{i}^{\dagger} f_{j} b_{j}^{\dagger} b_{i}-\frac{J}{2} \sum_{\langle i, j\rangle} b_{i} b_{j} b_{j}^{\dagger} b_{i}^{\dagger} \\
& \times\left(f_{i \uparrow}^{\dagger} f_{j \downarrow}^{\dagger}-f_{i \downarrow}^{\dagger} f_{j \uparrow}^{\dagger}\right)\left(f_{j \downarrow} f_{i \uparrow}-f_{j \uparrow} f_{i \downarrow}\right) .
\end{aligned}
$$

The above $U(1)$ slave-boson expression can also be deduced from our earlier $S U(2)$ slave-boson representation [33] of the $t-J$ Hamiltonian. The second term shows spinon pairing (spin singlet) interactions between adjacent sites. Both terms explicitly manifest coupling between the charge (holon) and spin (spinon) degrees of freedom.

We attempt a multi-faceted study of phase separation by employing various levels of approaches; the HatreeFock (HF) 33] of Hubbard Hamiltonian for a qualitative, comparative study of phase separations, the Monte Carlo diagonalization (MCD) 34] for an accurate study of correlated electron systems and the slave-boson functional integral (SBFI) method for an investigation of the role of charge (hole) and spin degrees of freedom on phase separation. Extending our earlier HF approach [33] of Hubbard Hamiltonian to a study of temperature dependence of charge and spin distributions in real space [35], we first examine the domain of phase separation in the plane of temperature vs. hole density and compare the 
phase separation domain derived from the Maxwell's construction with the one obtained from the direct (real) space calculations of electron-rich and hole-rich phases. Based on HF calculations with $U=4 t$, in Fig. 目 we display the predicted phase separation diagram in the plane of the temperature $T$ vs. hole density $x$ with the two different approaches; one from the use of the Maxwell's construction (denoted by shaded area in Fig. 11) and the other from the direct evaluations of hole (charge) and electron (spin) distributions (denoted as solid diamonds) in the real space of $14 \times 14$ lattice. Temperature dependent HF total energies for Maxwell's construction are obtained from the solution of the following self-consistent equations (Eq. (5)) involving the quasi-particle energy,

$$
E_{\mathbf{k}}^{ \pm}=\frac{U}{2}(1-x)-\mu \pm \epsilon_{\mathbf{k}}
$$

with $\epsilon_{\mathbf{k}}=\sqrt{\left[-2 t\left(\cos k_{x}+\cos k_{y}\right)\right]^{2}+\left(\frac{m U}{2}\right)^{2}}$ and $m=$ $\left|\left\langle c_{i \uparrow}^{\dagger} c_{i \uparrow}-c_{i \downarrow}^{\dagger} c_{i \downarrow}\right\rangle\right|$, by determining the uniform staggered magnetization $m$ and the chemical potential $\mu$

$$
\begin{aligned}
& 1=\frac{1}{N} \sum_{\mathbf{k}}^{\prime} \frac{U}{2 \epsilon_{\mathbf{k}}}\left[\tanh \left(\frac{E_{\mathbf{k}}^{+}}{2 T}\right)-\tanh \left(\frac{E_{\mathbf{k}}^{-}}{2 T}\right)\right] \\
& x=\frac{1}{N} \sum_{\mathbf{k}}^{\prime}\left[\tanh \left(\frac{E_{\mathbf{k}}^{+}}{2 T}\right)+\tanh \left(\frac{E_{\mathbf{k}}^{-}}{2 T}\right)\right] .
\end{aligned}
$$

Encouragingly the two results showed a large overlapping domain of phase separation in the $T-x$ plane. The present mean field results are at best qualitative.

In order to show the doping dependence of phase separation in real space, we now examine the electron and hole distributions interior and exterior to the phase separation boundary. At a chosen hole density of $x=0.25$ near but below a critical value $x_{c} \simeq 0.3$ at $T=0.01 t$, a stripe phase no longer appears and, instead, an inhomogeneous phase separation is revealed, as is shown in Fig. 2. Although not shown here, such inhomogeneous structures are prevalent near but below the critical hole density $x_{c}$, but they eventually disappear to yield uniform phase structures beyond $x_{c}$. It should be noted that despite the fact that no correlations exist between the Hatree-Fock quasiparticles, the phase separation is seen to occur as well known. They are not accurate for strongly correlated (large $U$ ) electron systems. However, it is to be noted that with currently available, numerically exact methods, the present level of real space calculations of temperature dependent phase separation for a large square lattice are not easily feasible. It is of great interest to see whether there exists a possibility of 'metastable' phase-separation in a certain region of temperature and doping where density fluctuations are excessively large as is shown by the results from the Maxwell's construction. This case happened near $T=0.01 t$ and $x=0.25$, showing a metastable region of phase separation. In this small domain, the density fluctuations were predicted to be large. A detailed study for verification is necessary in the future.

For an accurate account of strongly correlated electrons for the study of phase separation as a function of antiferromagnetic coupling strength $J$, we now take the MCD method [34] of the $t-J$ Hamiltonian, Eq. (1) with $J=1.0 t$ for a $4 \times 4$ square lattice. Fig. 3 shows the computed ground state energy (in units of $t$ ) shifted by a linear factor $e_{H} n_{e}$ as a function of the electron density $n_{e}\left(n_{e}=1-x\right)$. The solid triangle represents the GFMC calculation by Hellberg and Manousakis 25. ${ }^{c}$ and the open circles, our present results. From the Maxwell's construction the critical electron density (hole density) is predicted to be $n_{c}=0.684\left(x_{c}=0.316\right)$ compared to the value of $n_{c}=0.730\left(x_{c}=0.270\right)$ obtained from the GFMC. The overall variation of curvature as a function of electron (hole) density is grossly similar between the two approaches, although the predicted ground state energies are not the same. In Fig. A, we display our MCD predicted phase separation boundary in the plane of electron (hole) density vs. antiferromagnetic interaction strength (Heisenberg coupling constant) in units of $t$ and make comparison with other methods. The MCD agreed very well with the GFMC of Hellberg and Manousakis [25 ${ }^{c}$, the LED of Emery et al. [15] and the SBFI $(U(1)$ slave-boson functional integral) result of Gimm and Salk 30]. A salient feature is that all of these methods yielded a similar phase separation boundary, by showing a smoothly decreasing (increasing) trend of the critical electron (hole) density with an intercept near $J / t \sim 3.5$ as the antiferromagnetic interaction strength $J / t$ increases.

However, the accurate MCD calculations can not readily resolve the current controversy over the issue of whether the effect of correlations between the hole pairs is the primary cause of forming the hole-rich phase. For this cause we now explore the $U(1)$ slave-boson functional integral approach of the $t-J$ Hamiltonian (Eq. (3)). This method is advantageous to examine the role of the charge and spin degrees of freedom or the role of the holon and spinon degrees of freedom on phase separation. Earlier we reported that this method [30] also showed a satisfactory phase separation boundary for all $J$ values $(J / t \leq 1$ and $J / t>1$ ) in general agreement with other numerical studies [15,25]. The spin (spinon) degrees of freedom shown in the second term in Eq. (3) allows spinon pairing (spin singlet formation) interactions between adjacent sites. Indeed it has been shown from the $U(1)$ slave-boson theories that the spinon pairing, that is, the spin singlet pair order appears below the pseudogap (spin gap) temperature $T^{*} 36,33$. This indicates that the motion of paired holes rather than the independent motion of separated holes is energetically preferred in the presence of surrounding electron spin singlet pairs (spinon pairs) below the pseudogap (spin gap) temperature $T^{*}$. 
This is because the hole pairs (holon pairs) can readily migrate to the occupied sites of spin pairs (spinon pairs) involving no spin-bond breaking.

In summary, we showed that the phase separation diagram obtained from Maxwell's construction in the plane of temperature vs. hole density is consistent with one derived from the real space (direct lattice) calculations of hole-rich and electron-rich phases; inhomogeneous phase separation appears near $x_{c}$ at finite temperatures; the formation of the hole-rich phase for phase separation is attributed to the aggregation of hole pairs induced by spin singlet pairs which exist in the spin-gap phase.

One of us (S.H.S.S.) greatly acknowledges generous supports of POSRIB (2001) project at Pohang University of Science and Technology and Korean Ministry of Education (Hakjin Excellence Leadership Program 2001). We thank Chan-Ho Yang for computational assistances.

[1] M. v. Zimmermann, A. Vigliante, T. Niemöller, N. Ichikawa, T. Frello, J. Madsen, P. Wochner, S. Uchida, N. H. Andersen, J. M. Tranquada, D. Gibbs, and J. R. Schneider, Europhys. Lett. 41, 629 (1998).

[2] J. M. Tranquada, J. D. Axe, N. Ichikawa, A. R. Moodenbaugh, Y. Nakamura, and S. Uchida, Phys. Rev. Lett. 78, 338 (1997); J. M. Tranquada, J. D. Axe, N. Ichikawa, Y. Nakamura, S. Uchida, and B. Nachumi, Phys. Rev. B. 54, 7489 (1996); J. M. Tranquada, B. J. Sternlieb, J. D. Axe, Y. Nakamura, and S. Uchida, Nature 375, 561 (1995); P. Wochner, J. M. Tranquada, D. J. Buttrey, and V. Sachan, Phys. Rev. B, 57, 1066 (1998); J. M. Tranquada, D. J. Buttrey, V. Sachan, and J. E. Lorenzo, Phys. Rev. Lett. 73, 1003 (1994); V. Sachan, D. J. Buttrey, J. M. Tranquada, J. E. Lorenzo, and G. Shirane, Phys. Rev. B. 51, 12742 (1995); J. M. Tranquada, J. E. Lorenzo, D. J. Buttrey, and V. Sachan, ibid. 52, 3581 (1995).

[3] A. Lanzara, P. V. Bogdanov, X. J. Zhou, S. A. Kellar, D. L. Feng, E. D. Lu, T. Yoshida, H. Elsaki, A. Fusimori, K. Kishio, J.-I. Shhimoyama, T. Noda, S. Uchida, Z. Hussain, and Z. X. Shen, Nature 412, 510 (2001); references therein.

[4] J. D. Jorgensen, B. Dabrowski, S. Pei, D. G. Hinks, L. Soderholm, B. morosin, J. E. Schriber, E. L. Venturini, and D. S. Ginley, Phys. Rev. B 38, 11337 (1988).

[5] M. F. Hundley, J. D. Thompson, S-W. Cheong, Z. Fisk, and J. E. Schirber, Phys. Rev. B 41, 4062 (1990).

[6] S.-H. Lee and S-W. Cheong, Phys. Rev. lett. 79, 2514 (1997); P. C. Hammel, A. P. Reyes Z. Fisk, M. Takigawa, J. D. Thompson, R. H. Heffner, S-W. Cheong, and J. E. Schirber, Phys. Rev. B, 42, 6781 (1990); P. C. Hammel et al., Physica C 185-189, 1095 (1991).

[7] A. Weidinger, Ch. Niedermayer, A. Golnik, R. Simon, and E. Recknagel, Phys. Rev. Lett. 62, 102 (1989).

[8] D. R. Harshman, G. Aeppli, B. Batlogg, G. P. Espinosa, R. J. Cava, A. S. Cooper, L. W. Rupp, E. J. Ansaldo, and D. Ll. Williams, Phy. Rev. Lett. 63, 1187 (1989).

[9] J. H. Cho, F. Borsa, D. C. Johnston, and D. R. Torgeson, Phys. Rev. B 46, 3179 (1992); J. H. Cho, F. C. Chou, and D. C. Johnston, Phys. Rev. Lett. 70, 222 (1993).

[10] F. Borsa, P. Carretta, A. Lascialfari, D. R. Torgeson, F. C. Chou, and D. C. Johnston, Physica C 235-240, 1713 (1994); F. Borsa, P. Carretta, J. H. Cho, F. C. Chou, Q. Hu, D. C. Johnston, A. Lascialfari, D. R. Torgeson, R. J. Gooding, N. M. Salem, and K. J. E. Vos, Phys. Rev. B 52, 7334 (1995); F. C. Chou and D. C. Johnston, Phys. Rev. B 54, 572 (1996).

[11] X. L. Dong, Z. F. Dong, B. R. Zhao, Z. X. Zhao, X. F. Duan, L.-M. Peng, W. W. Huang, B. Xu, Y. Z. Zhang, S. Q. Guo, L. H. Zhao, and L. Li, Phys. Rev. Lett. 80, 2701 (1998).

[12] A. W. Hunt, P. M. Singer, K. R. Thurber, and T. Imai, Phys. Rev. Lett. 82, 4300 (1999); references therein.;

[13] E. G. Nikolaev, H. B. Brom, and A. A. Zakharov, Phys. Rev. B 62, 3050 (2000).

[14] J. Zaanen and O. Gunnarsson, Phys. Rev. B 40, 7391 (1989); J. Zaanen and P. B. Littlewood, Phys. Rev. B. 50, 7222 (1994).

[15] V. J. Emery, S. A. Kivelson, and H. Q. Lin, Phys. Rev. Lett. 64, 475 (1990); S. A. Kivelson, V. J. Emery, and H. Q. Lin, Phys. Rev. B 42, 65223 (1990); S. A. Kivelson and V. J. Emery, in Strongly Correlated Electronic Matrials: the Los Alamos Symposium, 1993, edited by K. S. Bedel et al. (Addison-Wesley, Reading, MA, 1994); V. J. Emery and S. A. Kivelson, Physica 209C, 597 (1993).

[16] G. Seibold, C. Castellani, C. Di Castro, and M. Grilli, Phys. Rev. B 58, 13506 (1998).

[17] M. U. Luchini, M. U. Ogata, W. O. Puttika, and T. M. Rice, Physica 185-189C, 141 (1991); W. O. Putikka, M. U. Luchini, and T. M. Rice, Phys. Rev. Lett. 68, 538 (1992).

[18] S. R. White, Phys. Rev. Lett. 69, 2863 (1992); Phys. Rev. B 48, 10345 (1993).

[19] W. O. Putikka, M. U. Luchini, and T. M. Rice, Phys. Rev. Lett. 68, 538 (1992).

[20] E. Dagotto, Rev. Mod. Phys. 66, 763 (1994); H. Fehske, V. Waas, H. Röder, and H. Bütter, Phys. Rev. B 44, 8473 (1991); D. poilblanc, ibid. 52, 9201 (1995).

[21] P. Prelovšek and X. Zotos, Phys. Rev. B 47, 5984 (1993).

[22] C. Castellani, C. Di Castro, and M. Grilli, Phys. Rev. B 40, 7391 (1989).

[23] D. Poliblanc, Phys. Rev. B 52, 9201 (1995).

[24] M. Kohno, Phys. Rev. B 55, 1435 (1997).

[25] a) C. S. Hellberg and E. Manousakis, Phys. Rev. Lett. 78, 4609 (1997); b) J. Phys. Chem. Solids 59, 1818 (1998); c) Phys. Rev. B 61, 11787 (2000).

[26] B. Shraiman and E. Siggia, Phys. Rev. Lett. 60, 740 (1988); 61, 467 (1988); M. Boninsegni and E. Manousakis, Phys. Rev. B 45, 4877 (1992); 46, 560 (1992).

[27] M. Calendra, F. Becca, and S. Sorella, Phys. Rev. Lett. 81, 5185 (1998).

[28] L. P. Pryadko, S. Kivelson, and D. W. Hone, Phys. Rev. Lett. 80, 5651 (1998).

[29] A. C. Cosentini, M. Capone, L. Guidoni and G. B. Bachelet, Phys. Rev. B 58, R 14685 (1998).

[30] T. H. Gimm and Sung-Ho Suck Salk, Phys. Rev. B 62, 
13930 (2000).

[31] S. R. White and D. J. Scalapino, Phys. Rev. Lett. 84, 3021 (2001); 80, 1272 (1998);81, 3227 (1998); Phys. Rev. B 60, 753 (1999).

[32] S. Hellberg and E. Manousakis, Phys. Rev. lett. 84, 3022 (2001); 83, 132 (1999).

[33] S.-S. Lee and Sung-Ho Suck Salk, Phys. Rev. B 64, 052501 (2001); T.-H. Gimm, S.-S. Lee, S.-P. Hong and Sung-Ho Suck Salk, Phys. Rev. B 60, 6324 (1999).

[34] H. De Raedt and W. von der Linden, Int. J. Mod. Phys. C 3, 97 (1992); Phys. Rev. B 45, 8787 (1992); W. von der Linden, Phys. Rep. 220, 53; H. De Raedt and M. Frick, Phys. Rep. 231, 107 (1993);

[35] S.-P. Hong, S.-S. Lee, and Sung-Ho Suck Salk, Phys. Rev. B 62, 14880 (2000).

[36] G. Kotliar and J. Liu, Phys. Rev. B 38, 5142 (1988); M. U. Ubbens and P. A. Lee, Phys. Rev. B 46, 8434 (1992); 49, 6853 (1994); X. G. Wen and P. A. Lee, Phys. Rev. Lett. 76, 503 (1996); 80, 2193 (1998); N. Nagaosa and P. A. Lee, Phys. Rev. B 61, 9166 (2000).

\section{FIGURE CAPTIONS}

FIG. 1 The temperature (in the unit of hopping energy $t$ ) dependence of phase separation as a function of hole doping density $x$ based on the Hatree-Fock approximation with $U=4 t$ for a $14 \times 14$ square lattice. The shaded region represents phase separation obtained from the Maxwell's construction; the solid diamond, the phase separation obtained from the numerical evaluation of charge and spin distributions in real space and the open diamond, the uniform phase.

FIG. 2 Spin (electron) and charge (hole) distribution at $x=50 / 196(\simeq 0.25) T=0.01 t$. The large circles represent holes (charges) and smaller ones, electrons (spins). The size of circles represents the degree of hole-richness or electron-richness.

FIG. 3 Maxwell's construction (dotted line) from predicted ground state energies denoted by open circles; the energies (in units of $t$ ) are shifted by a linear factor $-e_{H} n_{e},\left(e_{h}(x)-e_{H} n_{e}\right) / t$ as a function of electron density for $J=1.0 t$ with a $4 \times 4$ lattice. For comparison the Green's function Monte Carlo result [25] (denoted by HM) with various lattice sizes up to $11 \times 11$ is displayed.

FIG. 4 Phase separation for the hole-doped systems of antiferromagnetically correlated electrons in the plane of Heisenberg coupling strength, $J / t$ and the electron density, $n_{e}=1-x$. The triangles denoted by HM represent the Green's function Monte Carlo prediction of Hellberg and Manousakis 25 $]^{c}$ and the stars denoted by EKL, the exact diagonalization result of Emery et al. |15]. The solid circles represent the results from the $U(1)$ slave-boson functional integral approach of Gimm and Salk 30] and diamonds, our present MCD results. 


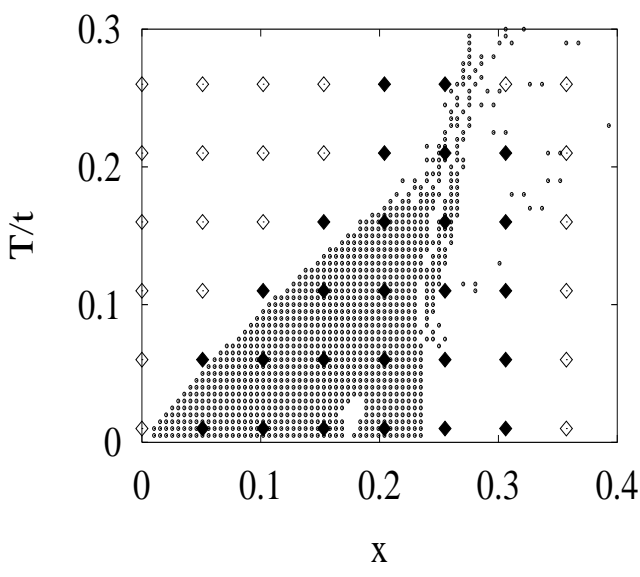

FIG. 1.

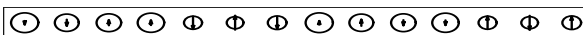

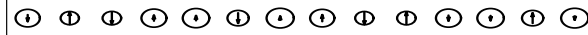
(1) $\quad \Phi \quad \Phi \quad 1(1)$

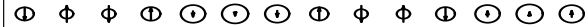
(1) (1) 1 () $\odot$ (1) $\odot$ (1) (1)

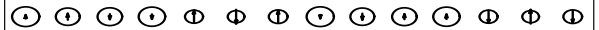

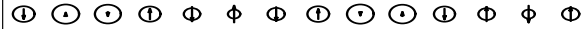

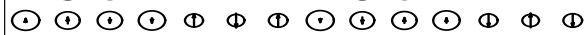

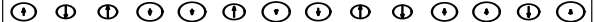
(1) $\quad \Phi \quad \Phi \quad 1(1)$

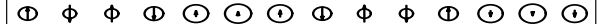
(1) (1) (1) $\odot$ (1) () (1) (1)

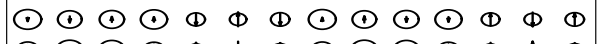

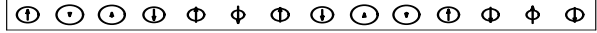

FIG. 2.

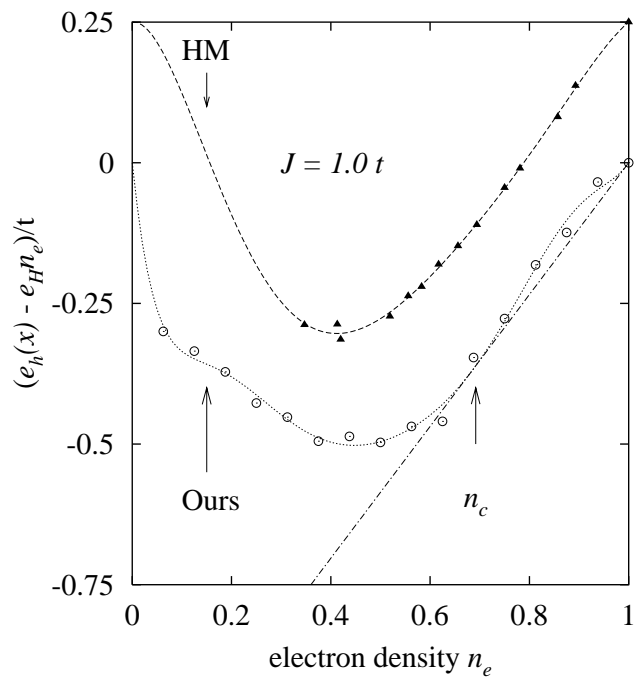

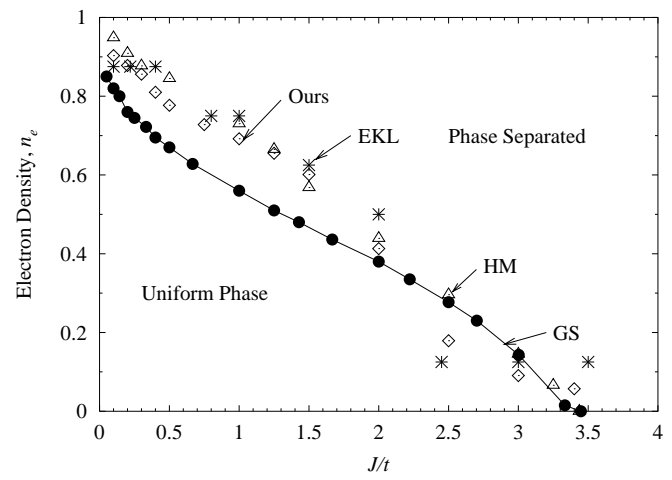

FIG. 4.

FIG. 3. 